\title{
Comparison of Lipid Lowering Effect of Aqueous Extract of Cinnamon (Cinnamomum Cassiae) with that of Rosuvastatin on Experimentally Induced Hypercholesterolaemic Rats
}

\author{
Sagia Afrose, ${ }^{1}$ Md. Ismail Khan, ${ }^{2}$ Elisa Omar Eva, ${ }^{3}$ Mohammad Imtiaj Mahbub ${ }^{4}$
}

\begin{abstract}
Background: Hypercholesterolemia is a condition characterized by very high level of cholesterol in the blood. Too much cholesterol increases risk of developing heart disease called coronary artery disease. This condition occurs when excess cholesterol in the bloodstream is deposited in the walls of blood vessels, particularly in the coronary arteries that supply blood to the heart. A phenolic compound of the cinnamon extract lowers serum lipid level by inhibiting HMG COA reductase $\&$ by activation of nuclear receptor peroxisome proliferator activated receptor alpha (PPAR- $\alpha$ ). We are trying to evaluate the lipid lowering effect of cinnamon (Cinnamomum cassiae) in comparison with rosuvastatin in hypercholesterolaemic rats.
\end{abstract}

Aim: To find out the lipid lowering effect of aqueous extract of cinnamon (Cinnamomum cassiae) and compare it with a established lipid lowering drug (rosuvastatin) on hypercholesterolaemic rats.

Method: This was a experimental study conducted in the department of Pharmacology, Dhaka medical college \& Hospital from July 2015 to June 2016. Sample size was 30.The study was designed as 2 parts: Experiment-1 \& Experiment -2.

Result: Cinnamon produces no statistically significant effect on serum lipid level of healthy rats ( $P$ - value $>0.05$; which is not significant). Cinnamon significantly reduced serum lipid level of high cholesterol diet induced hypercholesterolaemic rats. But, there is statistically significant difference between cinnamon and rosuvastatin lipid lowering effect in high cholesterol diet induced hypercholesterolaemic rats ( $p$ value $<0.05$; which is significant).

Conclusion: The study was conducted to find out lipid lowering effect of cinnamon on experimentally induced hypercholesterolaemic rats. The present study found that cinnamon (Cinnamomum cassaiae) significantly lowers serum lipid level in experimentally induced hypercholesterolaemic rats. So cinnamon can be used as alternative lipid lowering agent for its easy availability, cost effectiveness and as well as lack of significant side effects.

Key words: Hypercholesterolaemic, Cinnamon, Rosuvastatin, High cholesterol diet (HCD)

TAJ 2018; 31: No-1: 52-61

\section{Introduction}

Hyperlipidaemia may be defined as higher lipid or cholesterol level in the serum due to dietary or idiopathic condition. ${ }^{1}$ It is traditionally defined as a condition in which the concentration of cholesterol or triglyceride carrying lipoproteins in

${ }^{1}$ Assistant Professor, Department of Pharmacology, Bashundhara Ad-din Medical College, South Keranigonj, Dhaka

2 Vice Chancellor, Medical University of Chittagong, Chittagong

3 Professor \& Head, Department of Pharmacology, Shaheed Suhrawardy Medical College, Dhaka

${ }^{4}$ Assistant Professor, Department of Endocrinology, Rajshahi Medical College \& Hospital, Rajshahi 
plasma exceed an arbitrary normal limit. Hyperlipidaemia arise from the interplay between genetic predisposition \& diet, or they may be secondary to another condition such as hypothyroidism, nephrosis, obstructive liver disease, alcoholism, myeloma, pregnancy etc. ${ }^{2}$ Cholesterol is a waxy, fat like substance that is produced in the body \& obtained from food that come from animals (particularly egg yolk, meat, poultry, fish, dairy products). Body need the substance to build cell membranes, make certain hormones, \& produce compounds that aid in fat digestion. $^{3}$ Clinical concern arise because an elevated concentration of lipoproteins can accelerate the development of atherosclerosis, with its dual sequel of thrombosis \& infarction. This condition occurs when excess cholesterol in the blood stream is deposited in the wall of blood vessel, particularly the artery that supplies blood to the heart. The abnormal buildup of cholesterol forms clumps that narrow \& harden the artery walls. As the clumps get bigger, they can clog the arteries \& restricts the flow of blood to the heart. The buildup of plaque in the coronary arteries causes a form of chest pain called angina \& greatly increase a person's risk of having a heart attack. Inherited forms of hypercholesterolemia can also cause death problems related to the buildup of excess cholesterol in other tissues. If cholesterol accumulated in tendons, it causes characteristic growths called tendon xanthomas. These growths often affect the Achilles tendons \& tendons of hands \& fingers. Yellowish cholesterol deposits under the skin of eyelids known as xanthelasmata. Cholesterol can also accumulated in the cornea, leading to a gray colored ring called arcus cornealis. ${ }^{4}$ Recently, hypercholesterolemia has been associated with enhanced oxidative stress related to increase lipid peroxidation. ${ }^{5}$ Many studies indicate that lowering the serum cholesterol may prevent, control \& even reverse atherosclerosis \& coronary heart disease. Modern oral lipid lowering drugs are costly and are to be continued lifelong. They have adverse effects as well. ${ }^{6}$ Plants have been used for treatment of dyslipidaemia since $1550 \mathrm{BC}^{7}$ Spices have been used since ancient times not only for increasing the flavour of foods but also for their preservative and medicinal properties. ${ }^{8} \mathrm{~A}$ number of spices and herbs have a long history of traditional use in treating elevated blood sugar levels. Cinnamon (Cinnamomum cassiae) is one of the traditional folk herbs used for treatment of hypercholesterolemia. It lowers triacylglycerol \& low density lipoprotein cholesterol levels are desirable health outcomes. ${ }^{9}$

With this background information, in the present study, it was attempted to compare the lipid lowering effect of cinnamon (Cinnamomum cassiae) with an established lipid lowering drug (Rosuvastatin) in experimentally induced hypercholesterolaemic rats.

\section{Materials and Methods}

This was an experimental study conducted in the department of Pharmacology, Dhaka medical college \& Hospital from July 2015 to June 2016. Sample size was 30.The study was designed as 2 parts: Experiment-1 \& Experiment-2. The experiment was carried out on a total number of 30 long Evans Norwegian strain rats of both sex, weighing between 140-150 gram and age between 8-10 weeks. Total numbers of rats are divided into 5 groups- Group (A-E). Each group contained 6 numbers of rats. Group (A, C) used as control group \& group (B, D, E) used as experimental group. Cinnamon powder was obtained from cinnamon bark \& powder was produced by crushing sun dried cinnamon bark in a grinder machine. Aqueous extract of Cinnamon was made in the Drug Research laboratory of Center for advanced research of sciences (CARS) of Dhaka University. Serum lipid profile estimation was done by enzymatic colorimetric (CHOD-PAP) method. Data was analyzed with the help of a computer by a statistical program SPSS. Unpaired "t" test was applied for data analysis and results were expressed as mean $\pm \mathrm{SE} \&$ values of probability $\mathrm{p}<0.05 \& \mathrm{p}>0.05$ will be considered to be statistically significant \&not significant respectively. 


\section{Experiment- 1}

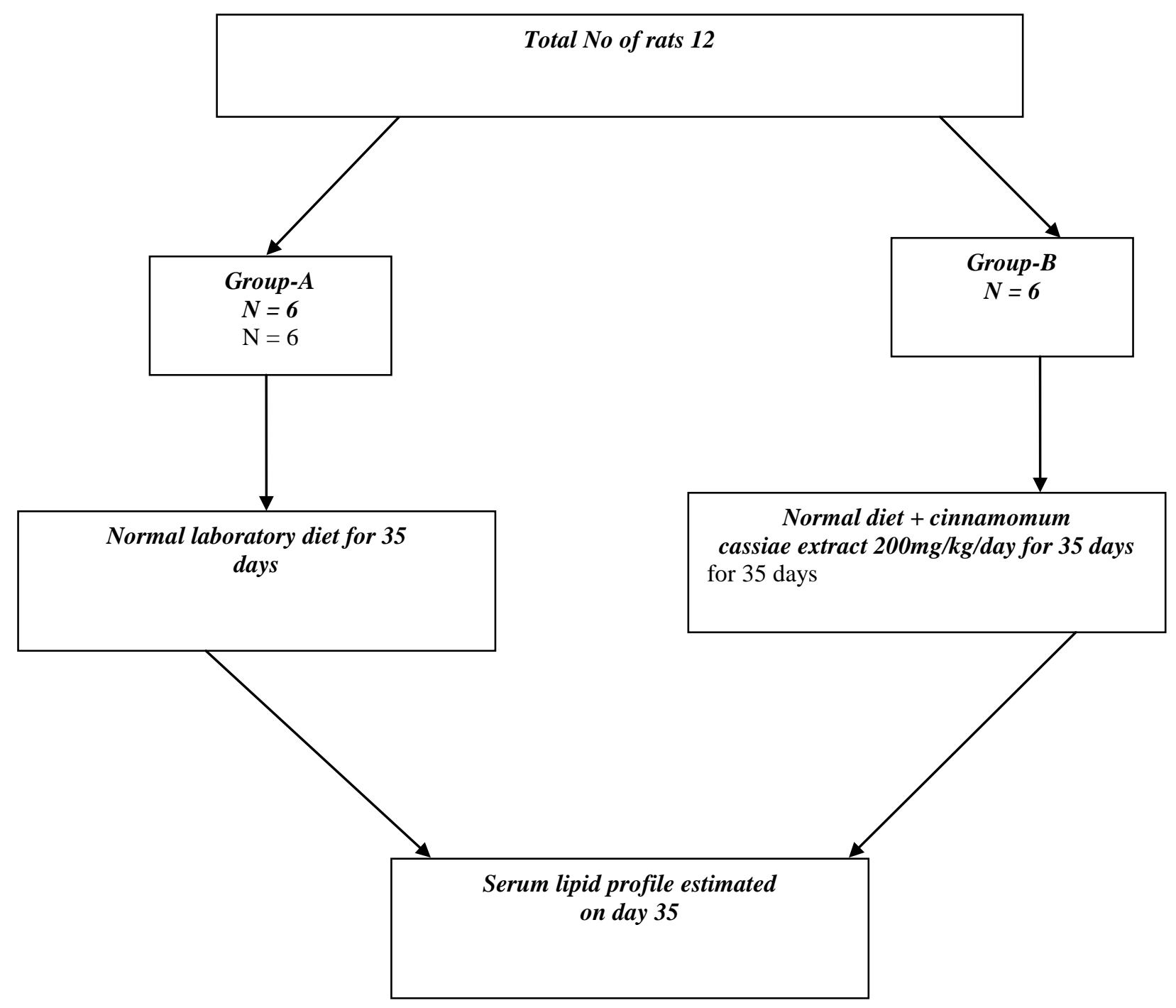

Design of experiment: 1 


\section{Experiment- 2}

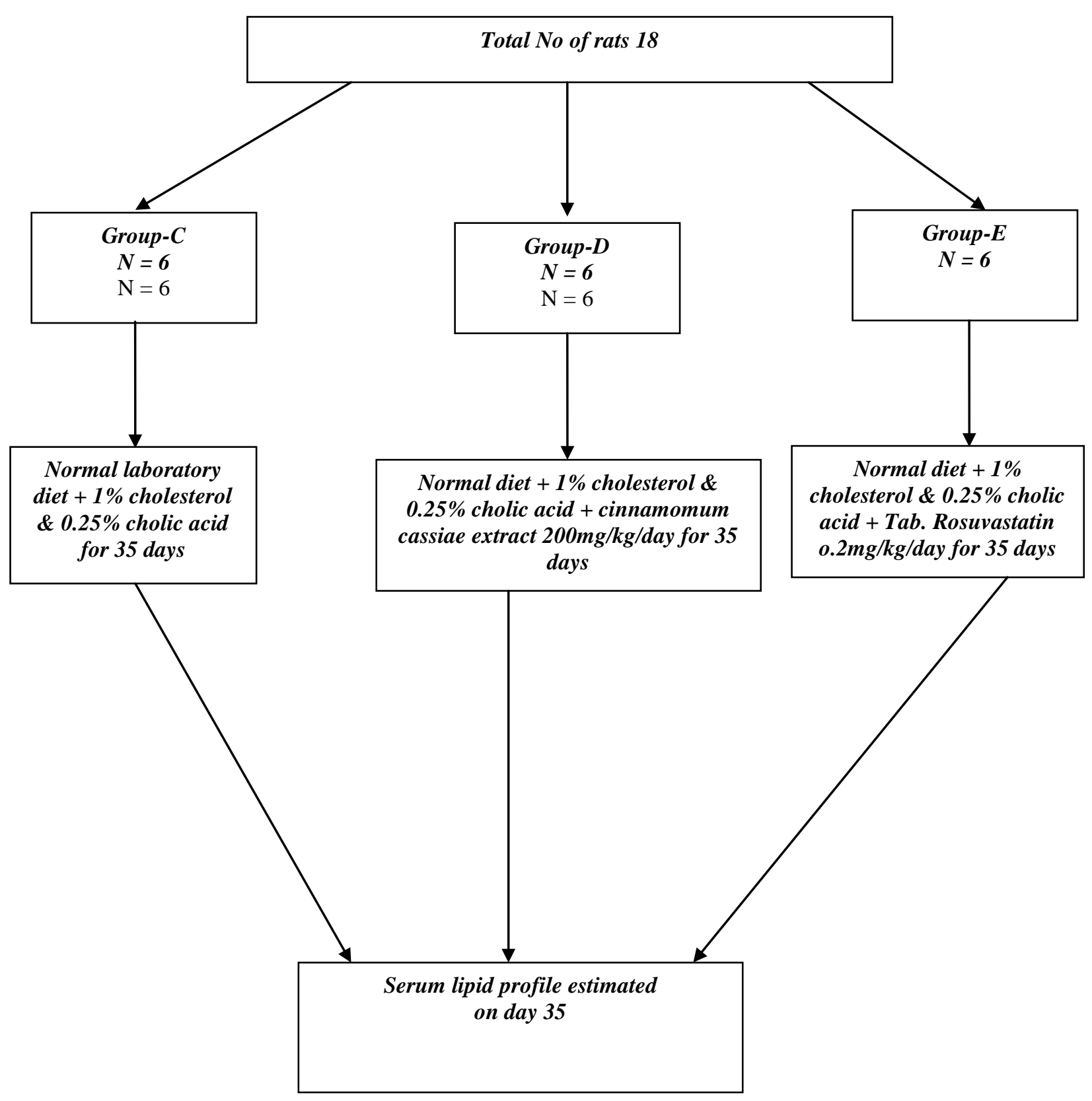

Design of experiment: 2 


\section{Results}

Table - l: Effects of aqueous extract of cinnamon (Cinnamomum cassiae) on serum lipid profile of adult rats with normal diet $(\mathrm{A} \infty \mathrm{B})$

\begin{tabular}{|c|c|c|}
\hline Group Control Group (A) & Cinnamon & p - value \\
\hline
\end{tabular}

Serum

Cholesterol conc. (mg/dl)71.17 $\pm 1.59 \quad 69.83 \pm 3.04 \quad 0.125^{\mathrm{ns}}$

$($ Mean \pm SE) $(n=6)$

Serum

TG conc. (mg/dl)

$78.10 \pm 2.45$

$76.33 \pm 1.93$

$0.21^{\text {ns }}$

$($ Mean \pm SE) $(n=6)$

Serum

HDL cholesterol conc. $31.50 \pm 0.93 \quad 30.67+4.87$

$0.11^{\mathrm{ns}}$

$(\mathrm{mg} / \mathrm{dl})($ Mean \pm SE) $(\mathrm{n}=6)$

Serum

LDL cholesterol conc.26.61 $\pm 2.23 \quad 29.90 \pm 6.00 \quad 0.089^{\mathrm{ns}}$

$(\mathrm{mg} / \mathrm{dl})($ Mean $\pm \mathrm{SE})(\mathrm{n}=6)$

Table I shows cinnamon produces no statistically significant effect on serum lipid profile of normal adult rats ( $\mathrm{p}$ value $>0.10$; which is not significant).

Group $-\mathrm{A}=$ Standard normal diet was given for 35 days

Group - B = Normal diet + Aqueous extract of cinnamon 200mg/kg/day was given for 35 days

Results were obtained through unpaired student's “ $t$ ” test

S.E = standard Error

$\mathrm{s}=$ statistically significant $(\mathrm{p}<0.05)$

ns = statistically not significant $(\mathrm{p}>0.05)$ 
Table II: Effects of Fatty mixture feeding on serum lipid profile of adult rats $(\mathrm{A} \propto \mathrm{C})$

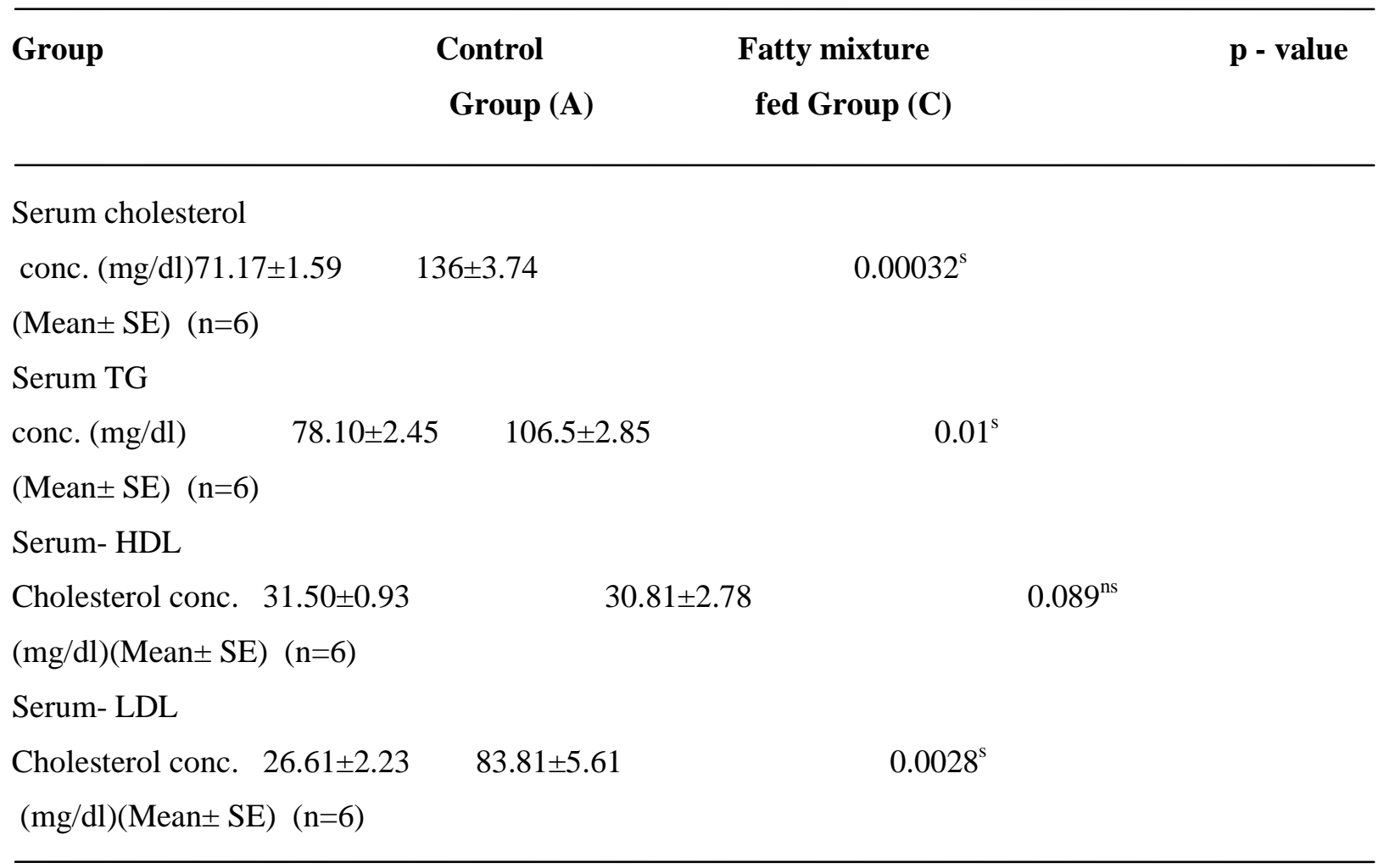

Table II shows high cholesterol diet significantly elevate the serum Cholesterol, TG-Cholesterol \& LDLCholesterol level (p- value $<0.05$; which is statistically significant) with no significant effect on serum HDL-Cholesterol level( $\mathrm{P}$ - value $>0.10$; which is not significant) of group $\mathrm{C}$ rats at day 35.

Group $-\mathrm{A}=$ Standard normal diet was given for 35 days

Group $-\mathrm{C}$ = was given normal laboratory diet $+1 \%$ cholesterol $\& 0.25 \%$ cholic acid for 35 days

Results were obtained through unpaired student's “ t” test

S.E = Standard Error

$\mathrm{s}=$ statistically significant $(\mathrm{p}<0.05)$

$\mathrm{ns}=$ statistically not significant $(\mathrm{p}>0.05)$ 


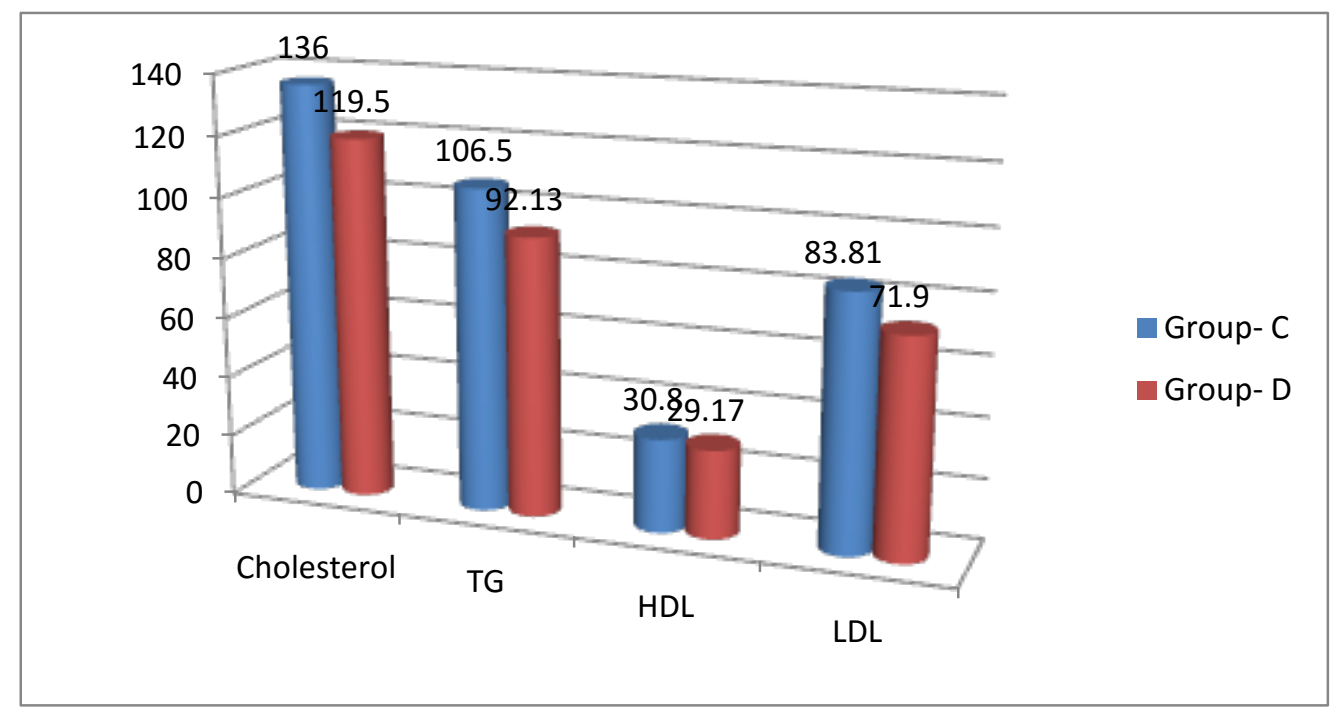

Group - C rats were given normal laboratory diet $+1 \%$ cholesterol \& $0.25 \%$ cholic acid for 35 days

Group - D rats were given normal diet $+1 \%$ cholesterol \& $0.25 \%$ cholic acid + Cinnamomum cassiae extract $200 \mathrm{mg} / \mathrm{kg} / \mathrm{day}$ for 35 days

Result were obtained through unpaired student's " $\mathrm{t}$ ” test

Figure A : Bar diagram showing that aqueous extract of cinnamon (Cinnamomum cassiae) significantly lowers the serum level of Cholesterol, TG \& LDL- Cholesterol on experimentally induced hypercholesterolaemic rats ( $\mathrm{P}$ - value $<0.05$; which is statistically significant) without significant effects on serum HDL-Cholesterol level ( $\mathrm{P}$ - value $>0.10$; which is not significant) comparing in group C \& D rats.

Table III: Effects of Rosuvastatin serum lipid profile of adult rats fed with fatty mixture fed $\quad$ (C $\infty$ E)

\section{Group Fatty mixture fed Rosuvastatin on fatty mixture fed \\ Group (C) Group (E) P p - value}

Serum Cholesterol

conc. (mg/dl)

$136 \pm 3.74 \quad 83.83 \pm 3.51$

$0.012^{\mathrm{s}}$

$($ Mean \pm SE $)(n=6)$

Serum TG

conc. (mg/dl)

$106.5 \pm 2.85 \quad 75.67 \pm 2.18$

$0.004^{\mathrm{s}}$

$($ Mean \pm SE $)(n=6)$

Serum- HDL

cholesterol conc. $30.81 \pm 2.78$

$30.50 \pm 2.09$

$0.134^{\text {ns }}$ 
$(\mathrm{mg} / \mathrm{dl})($ Mean \pm SE) $(\mathrm{n}=6)$

Serum- LDL

cholesterol conc. $83.81 \pm 5.61$

$(\mathrm{mg} / \mathrm{dl})($ Mean $\pm \mathrm{SE})(\mathrm{n}=6)$

Group $-\mathrm{C}=$ Standard normal diet was given for 35 days

Group $-\mathrm{E}=$ Normal diet $+1 \%$ cholesterol \& $0.25 \%$ cholic acid + Tab. Rosuvastatin $0.2 \mathrm{mg} / \mathrm{kg} /$ day was given for 35 days

Results were obtained through unpaired student's “t” test

Table III shows Rosuvastatin significantly reduced serum level of Cholesterol, TG \& LDL- Cholesterol on experimentally induced hypercholesterolaemic rats ( $\mathrm{P}$ - value $<0.05$; which is statistically significant) without significant effects on serum HDL-Cholesterol level ( $\mathrm{P}$ - value $>0.10$; which is not significant) comparing in group C \& E rats.

S.E = Standard Error

$\mathrm{s}=$ Statisticallysignificant $(\mathrm{p}<0.05)$

ns $=$ Statistically not significant $\quad(p>0.05)$

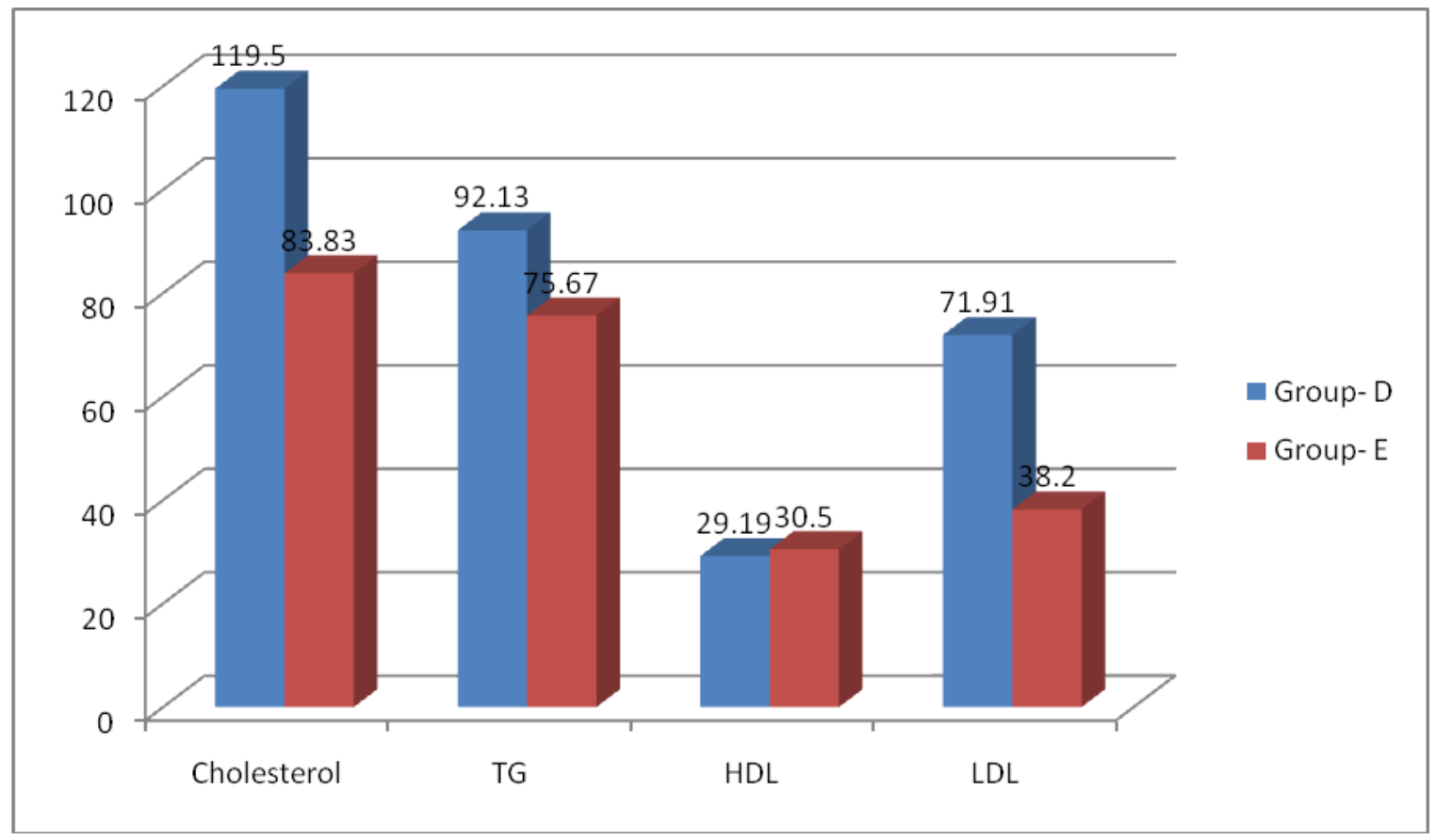

Group D rats were given normal diet $+1 \%$ cholesterol \& $0.25 \%$ cholic acid + Cinnamomum cassiae extract $200 \mathrm{mg} / \mathrm{kg} /$ day for 35 days

Group E rats were given normal diet $+1 \%$ cholesterol \& $0.25 \%$ cholic acid +Tab. Rosuvastatin $0.2 \mathrm{mg} / \mathrm{kg} /$ day for 35 days 
Result were obtained through unpaired student's " $\mathrm{t}$ " test

Figure B: Bar diagram showing the comparison between the lipid-lowering effects of aqueous extract of cinnamon (Cinnamomum cassiae) with that of Rosuvastatin on experimentally induced hypercholesterolaemic rats in group D \& E. There is statistically significant difference between cinnamon (Cinnamomum cassiae) \&Rosuvastatin on lipid lowering effect on experimentally induced hypercholesterolaemic rats ( $\mathrm{p}$ value $<0.05$; which is statistically significant)

\section{Discussion}

This was an experimental study, conducted in the department of pharmacology, Dhaka Medical College in between July, 2015 to June, 2016. The present study was carried out to evaluate the lipid lowering effect of cinnamon (Cinnamomum cassaiae) on experimentally induced diabetic rats. The blood glucose lowering effect of aqueous extract of cinnamon (Cinnamomum cassaiae) was tested in normal adult rats and experimentally induced hypercholesterolaemic rats. The aqueous extract of cinnamon (Cinnamomum cassaiae) was given for 35 days in normal rats and experimentally induced hypercholesterolaemic rats. The lipid lowering effect of cinnamon was compared with a standard oral lipid lowering drug, Rosuvastatin. This was in accordance with Lee al. ${ }^{10}$ where they also gave cinnamon 15 days in normal rats and 15 days in hypercholesterolaemic rats.

In the present study, hypercholesterolemia was induced by high cholesterol diet (1\% cholesterol \& $0.25 \%$ cholic acid). The dose of high cholesterol diet (HCD) was in similarity with Amin et al. ${ }^{11}$ The serum lipid profile of animals were measured 35 days after administration of HCD which was done according to the experiment of Adaramoy et al. $^{12}$

There was no statistically significant difference in serum lipid profile of normal rats treated with aqueous extract of cinnamon as compared with normal control ( $\mathrm{p}$ value $>0.05$, which is not significant). These findings are in consistent with Quin et al. ${ }^{13}$

In experiment-2, there was marked increase of serum lipid level by high cholesterol diet (HCD); which was also in similarity with Amin et al. ${ }^{11}$

Aqueous extract of cinnamon (Cinnamomum cassaiae) significantly reduces serum lipid profile in HCD induced hypercholesterolaemic rats (p value $>0.10$, which is significant). This study is in similarity with Adaramoye et al. $^{12}$ \& Lee et al. ${ }^{10}$ Thus this study hypothesis 'Aqueous extract of cinnamon (Cinnamomum cassaiae) has got lipid lowering effect in experimentally induced hypercholesterolaemic rats was established.

The possible mechanism of lipid lowering action of cinnamon extract may be a direct role in lipid metabolism \& prevent hypercholesterolemia \& lower free fatty acids by its strong lipolytic activity. Cinnamate, a phenolic compound found in the inner bark of the cinnamon lowers cholesterol level by inhibiting HMG-Co-A reductase Javed et al. ${ }^{14}$ also found same action of cinnamon.

In this study, cinnamon had got inferiority over rosuvastatin respect of lipid lowering effect on experimentally induced hypercholesterolaemic rats ( $p$ value $>0.10$, which is significant). This finding was not in similarity with Javed et al. ${ }^{14}$ where they found cinnamon has got almost similar lipid lowering effect like rosuvastatin. Larger sample size and longer duration of their study may explain this discrepancy.

\section{Conclusion}

The study was conducted to find out lipid lowering effect of cinnamon on experimentally induced hypercholesterolaemic rats. The present study found that cinnamon (Cinnamomum cassaiae) significantly lowers serum lipid profile in experimentally induced hypercholesterolaemic rats. So cinnamon can be used as alternative lipid lowering medicine for its easy availability, cost effectiveness and as well as lack of significant side effects. 


\section{Recommendation}

Further human study with large sample size and long duration is needed to find out the lipid lowering effect of cinnamon (Cinnamomum cassaiae) on dyslipidaemic patients.

\section{References}

1. Neville C.(2011) Oxford English Dictionary .12 th ed. London: Oxford university press.p.345.

2. Shoback C and David G. (2011) Dolores Greenspan's basic \& clinical endocrinology. New York: MacGraw-Hill Medical. $9^{\text {th }}$ ed.17.p.122.

3. Khan $A$, Safdar $M$, Khan MA, Khattack $K N$, Anderson RA.(2003) 'Cinnamon improves glucose and lipids of people with type 2 diabetes mellitus'. Diabetes Care.26.3215-3218.

4. Omonkhua A, Onoagbe I, Ajileye A, Aladegboye A.(2013) 'Long term anti diabetic, antihyperlipidaemic and anti atherogenic effects of carica papaya leaves in streptozotocin induced diabetic rats. European Journal of Medical Plants.4.p.508-519.

5. Kannappan S, Jayaraman T, Rajasekar P, Ravichandran MK., Anuradha CV. (2006)" Cinnamon bark extract improves glucose metabolism and lipid profile in fructose-fed rat' .Singapore Medical Journal. 10.p. 858-863.

6. Awal MA, Rashid MU, Ahmed KW. (1999) 'Effect of Kerala on lipid profile in hypercholesterolemic rats' Bangladesh J pharm .15.p.6-8.

7. Longe AD, Momah y, Adepoju PA.(2015) 'Effect of cinnamon aqueous extract on blood glucose level, liver biomarker enzymes, haematological and lipid profile parameters in alloxen- induced diabetic male albino rats' European Scientific Journal.1.p.1857-7431

8. Chi MS, Koh ET, Stewart AL. (1998) 'Effect of garlic on lipid metabolism in rats fed cholesterol'. J Nutr.112.p. 241-24 7.

9. Rahim A, Jamal AJ. (2009) 'Effects of cinnamon on blood glucose and lipid level in diabetic patients'. Jordan J of Biological Science. P.135-138.

10. Lee JS, Jeon SM, Park EM, Huh TL, Kwon OS, Lee MK9. (2003) 'Cinnamate supplementation enhances hepatic lipid metabolism and antioxidant defense systems in high cholesterol fed rat'. J. Medicinal Food .6(3).p.183--191.

11. Amin KA, AbdEL-Twab TM.(2009) 'Oxidative markers, nitric oxide and homocysteine alteration in hypercholesterolemic rats: role of atorvastatin and cinnamon'. Int J ClinExp Med .2(3).p 254-265.

12. Adaramoye, OA, Akintayo $\mathrm{O}$, Achem J, Fafunso MA.(2008) ' Lipid-lowering effects of methanolic extract of vernoniaamygdalina leaves in rats fed on high cholesterol diet'. Vascular health \& Risk Management . 4(1).p. 235--241.

13. Quin B., Nugasaki M., Ren M., Bijotto G.,Oshida Y.,sato Y.(2003) 'Cinnamon extract (traditional herb) potentates in vivo insulin regulated glucose utilization via enhancing insulin signaling in rats,. Diabetes Research and clinical practices. 62.p.139148.

14. Javed I., Faisal I, Khan MZ, Rahman Z, Muhammad F, Aslam B.(2012) ' Lipid lowering effect of cinnamomumzeylanicum in hyperlipidemic albino rabbit'. Pakistan J Pharm Sci . 25(1).p. 141-147 